\title{
Digital Ulcer as a Rare Complication of Systemic Lupus Erythematosus: A Case Report
}

\author{
Adem KÜÇÜK, ${ }^{1}$ Sinan BAĞCACI, ${ }^{2}$ Yalçın SOLAK, ${ }^{3}$ Recep TUNÇ ${ }^{1}$ \\ ${ }^{1}$ Division of Rheumatology, Department of Internal Medicine, Necmettin Erbakan University Meram School of Medicine, Konya, Turkey \\ ${ }^{2}$ Department of Physical Medicine and Rehabilitation, Necmettin Erbakan University Meram School of Medicine, Konya, Turkey \\ ${ }^{3}$ Division of Nephrology, Department of Internal Medicine, Necmettin Erbakan University Meram School of Medicine, Konya, Turkey
}

The two most common rheumatological diseases which accompany Raynaud's phenomenon are systemic sclerosis and systemic lupus erythematosus (SLE). Digital gangrene formation has no definitive etiology and pathogenesis, and Raynaud's phenomenon often accompanies the necrotic lesions associated with this skin condition. Macrovascular involvement is commonly found in systemic sclerosis, but is very rare in patients with SLE. In our case, we identified ulnar artery involvement in the absence of any other risk factors associated with digital gangrene formation. Raynaud's phenomenon is fairly common in SLE, but digital ulcers are seldomly seen. The most common cause of digital gangrene in SLE is antiphospholipid antibody syndrome (AAS). The risk factors for digital ulcers include duration of lupus, male gender, atherosclerosis, vasculitis, and AAS. Herein, we present a case with long-standing SLE who also had digital ulcers.

Keywords: Digital ulcer; gangrene; systemic lupus erythematosus.

Raynaud's phenomenon is an abnormal vasospasm of digital arteries, precapillary arterioles, and dermal arteriovenous shunts that occurs in response to cold and various other stimuli. ${ }^{1}$ Digital ulceration is commonly seen in connective tissue diseases, in particular systemic sclerosis. Although Raynaud's phenomenon is fairly common in patients with systemic lupus erythematosus (SLE), digital ulcers are quite rare. ${ }^{2}$ Herein, we report on a patient with long-term SLE who presented with digital ulcers.

\section{CASE REPORT}

A 45-year-old female patient who had a 10-year history of SLE presented to our outpatient rheumatology clinic with complaints of severe pain and cyanosis along with appearance of digital ulcers on her fingers (Figure 1). The patient had suffered from Raynaud's phenomenon in the past. She also had four living children and had experienced no miscarriages. Furthermore, the patient also had no diabetes, hypertension (HT), or heart disease, did not partake of alcohol, and was a nonsmoker. Her current medications at presentation included only oral methylprednisolone $4 \mathrm{mg} / \mathrm{day}$, and she appeared to be well and nondistressed. On physical examination, the patient's blood pressure was $110 / 70 \mathrm{mmHg}$, and her pulse was $80 \mathrm{bpm}$, and there were necrotic lesions in the distal parts of the index and fourth fingers of her right hand but no signs of cyanosis or edema. Moreover, the radial and ulnar pulses were weakly palpable on the right hand, her heart sounds were regular, and there were no audible murmurs. The other aspects of her physical examination were unremarkable. 


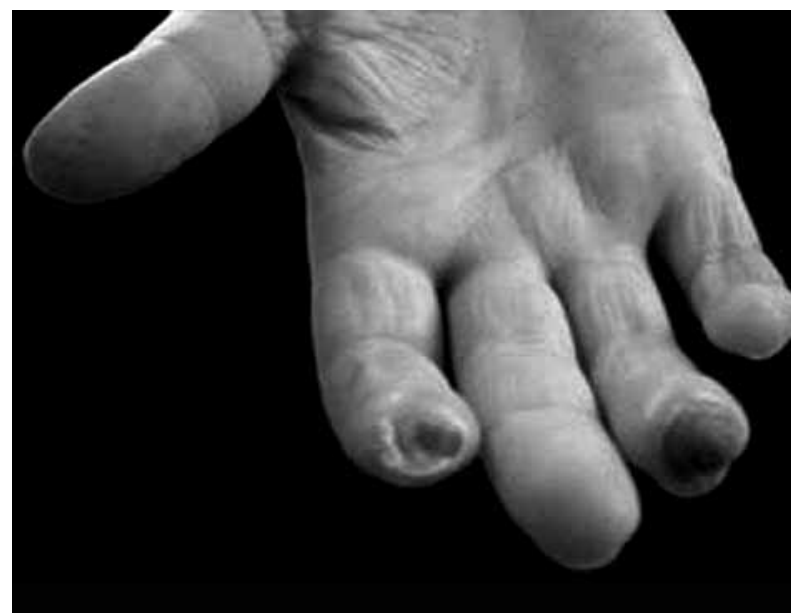

Figure 1. Necrotic ulcers in the index and fourth fingers of the right hand.

The biochemical values and blood counts were as follows: blood glucose: $76 \mathrm{mg} / \mathrm{dL}$, creatinine: $0.7 \mathrm{mg} / \mathrm{dL}$, alanine aminotransferase (ALT): $12 \mathrm{U} / \mathrm{L}$, low-density lipoprotein (LDL) cholesterol: $72 \mathrm{mg} / \mathrm{dL}$, triglycerides $105 \mathrm{mg} / \mathrm{dL}$, C-reactive protein (CRP): $11.7 \mathrm{mg} / \mathrm{L}$, white blood cell (WBC) count: $6700 / \mathrm{mm}^{3}$, hemoglobin: $9.6 \mathrm{~g} / \mathrm{dL}$, platelet count: $759 \times 10^{3}$, and erythrocyte sedimentation rate (ESR): $60 \mathrm{~mm} / \mathrm{h}$. Urinalysis showed pyuria with

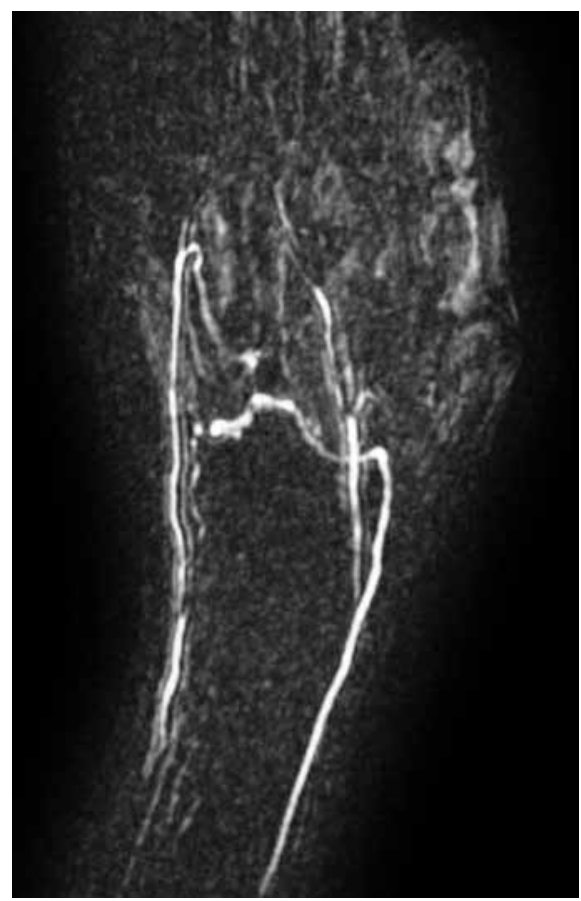

Figure 2. Occlusion and narrowness at the right ulnar artery at the right wrist level. no protein along with the presence red blood cells. The patient tested positive for antinuclear antibodies (1:640) at high titers in a homogeneous pattern, and for anti-double-stranded deoxyribonucleic acid (anti-dsDNA) antibodies at $35 \mathrm{IU} / \mathrm{ml}$ titers (normal $15 \mathrm{IU} / \mathrm{ml})$. In addition, the patient also tested positive for anti-SSA antibodies at $45 \mathrm{U} / \mathrm{ml}$ titers ( $220 \mathrm{U} / \mathrm{ml}$ : negative, $20-25 \mathrm{U} / \mathrm{ml}$ : borderline, and $>25 \mathrm{U} / \mathrm{ml}$ : positive). However, the urine culture yielded no growth, and the results for antineutrophil cytoplasmic antibodies (ANCAs), the Venereal Disease Research Laboratory (VDRL) test, anticardiolipin immunoglobulin $\mathrm{G}(\mathrm{IgG})$, IgM, and the lupus anticoagulant were all negative. Furthermore, serum protein electrophoresis showed a polyclonal gamma band, and the patient's prothrombin time and activated partial thromboplastic time (aPTT) as well as her complement 3 (C3) and C4 levels were normal.

An arterial duplex Doppler ultrasound of the upper extremities showed a bilateral weak monophasic flow in the radial and ulnar arteries, and magnetic resonance angiography (MRA) of the upper extremities revealed that the right ulnar artery was occluded and narrowed at the level of the wrist (Figure 2). However, the left arm vessels and proximal vasculature on the right side were normal.

We administered cyclophosphamide $50 \mathrm{mg}$ (three times a day), the sustained release form of nifedipine $30 \mathrm{mg}$ daily, and $150 \mathrm{mg}$ acetylsalicylic acid (ASA) daily, and the necrotic ulcers had healed completely by the third month of this treatment regimen (Figure 3). The oral cyclophosphamide treatment continued for at total of six months, and

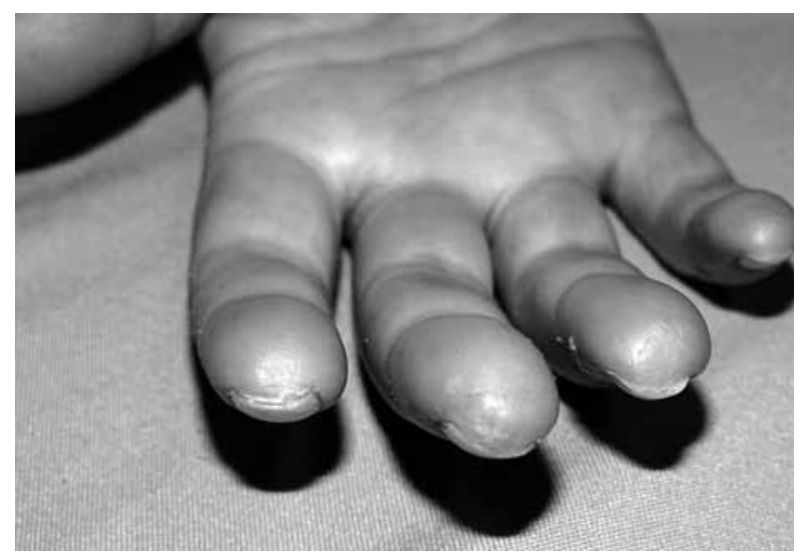

Figure 3. Healed ulcers at the third month of treatment. 
after that time, the patient had no active complaints. Since significant improvement had occurred, the cyclophosphamide was then discontinued, and the patient went on ASA and nifedipine for prophylaxis.

\section{DISCUSSION}

Despite being a common occurrence in connective tissue diseases, digital ulcer and gangrene development is only occasionally seen in patients with SLE. The most common cause of digital gangrene associated with SLE is antiphospholipid antibody syndrome (AAS), and SLE is the leading cause of secondary AAS. ${ }^{3}$ Several risk factors have been identified with digital ulcers in SLE, including duration of lupus, male gender, atherosclerosis, vasculitis, and AAS. ${ }^{4}$

Liu et al. ${ }^{5}$ demonstrated that duration of disease, the presence of Raynaud's phenomenon, and high serum CRP levels were determinants of digital ulcer development in a cohort of 2,600 lupus patients. When we looked at the risk factors in our patient, she had a 10-year history of lupus, but her disease activity was under control with small oral doses of corticosteroids. In addition, lupus has increasingly been recognized as a condition in which premature atherosclerosis develops, primarily because of increased, persistent inflammation. ${ }^{6,7}$ Our middle-aged patient had neither a prior history of heart disease nor a positive family history, and she was also a nonsmoker and a nondiabetic. Furthermore, peripheral arterial disease usually affects lower extremities, but our patient had no intermittent claudication, and the peripheral pulses in her lower extremities were bilaterally palpable. Moreover, the patient's CRP values were low, and her antiphospholipid antibodies were negative. The patient also had not experienced any previous or current vasculitis attacks. Hence, the only established risk our patient had for digital ulcer development was her history of Raynaud's phenomenon.

Gomes et al. ${ }^{8}$ evaluated digital vasculitis (DV) and other clinical findings according to the Systemic Lupus Erythematosus Disease Activity Index (SLEDAI) in 168 consecutive lupus patients. They also compared the SLEDAI scores of the both the patients with and without DV and found that those with DV had milder systemic involvement compared with the other patients. However, the SLEDAI system is known to assign a high score to patients with DV, and our case also supports this observation. Furthermore, despite the presence of digital gangrene, our patient had no major organ involvement at the time of presentation.

The treatment of digital ulcers in patients with SLE includes iloprost infusion, bosentan, and immunosuppressive therapy (cyclophosphamide and pulse methylprednisolone). ${ }^{9}$ Our patient responded well to six months of immunosuppressive oral cyclophosphamide and a combination of the calcium channel blocker nifedipine and low-dose ASA as an anti-platelet agent.

\section{Conclusion}

Digital ulcers may be seen in lupus patients as a part of DV, but as with our patient, they do not necessarily represent the presence of a severe systemic disease. Furthermore, our patient with DV responded positively to various treatment protocols, which bodes well for other patients with this condition.

\section{Declaration of conflicting interests}

The authors declared no conflicts of interest with respect to the authorship and/or publication of this article.

\section{Funding}

The authors received no financial support for the research and/or authorship of this article.

\section{REFERENCES}

1. Wigley FM. Clinical practice. Raynaud's Phenomenon. N Engl J Med 2002;347:1001-8.

2. Nagai $Y$, Shimizu A, Suto M, Tanaka S, Yasuda M, Tago $\mathrm{O}$, et al. Digital gangrene in systemic lupus erythematosus. Acta Derm Venereol 2009;89:398-401.

3. Mills JA. Systemic lupus erythematosus. N Engl J Med 1994;330:1871-9.

4. Drenkard C, Villa AR, Reyes E, Abello M, AlarcónSegovia D. Vasculitis in systemic lupus erythematosus. Lupus 1997;6:235-42.

5. Liu A, Zhang W, Tian X, Zhang X, Zhang F, Zeng X. Prevalence, risk factors and outcome of digital gangrene in 2684 lupus patients. Lupus 2009;18:1112-8.

6. Manzi S. Systemic lupus erythematosus: a model for atherogenesis? Rheumatology (Oxford) 2000;39:353-9.

7. Bhatt SP, Handa R, Gulati GS, Sharma S, Pandey RM, Aggarwal $P$, et al. Peripheral vascular disease in systemic lupus erythematosus. Lupus 2007;16:720-3. 
8. Gomes C, Carvalho JF, Borba EF, Borges CT, Vendramini $\mathrm{MB}$, Bueno C, et al. Digital vasculitis in systemic lupus erythematosus: a minor manifestation of disease activity? Lupus 2009;18:990-3.
9. Nagai Y, Shimizu A, Suto M, Tanaka S, Yasuda M, Tago $\mathrm{O}$, et al. Digital gangrene in systemic lupus erythematosus. Acta Derm Venereol 2009;89:398401. 